\title{
The Effects of Oral Ibuprofen on Medicinal Closure of Patent Ductus Arteriosus in Full-Term Neonates in the Second Postnatal Week
}

\author{
Mohammad Reza Alipour, ${ }^{1}$ Mansooreh Mozaffari Shamsi, ${ }^{1}$ Seyedeh Mahdieh Namayandeh, ${ }^{1}$ Zohreh \\ Pezeshkpour, ${ }^{1}$ Fatemeh Rezaeipour, ${ }^{1, *}$ and Mohammadtaghi Sarebanhassanabadi ${ }^{1}$ \\ ${ }^{1}$ Yazd Cardiovascular Research Center, Shahid Sadoughi University of Medical Sciences, Yazd, IR Iran \\ "Corresponding author: Fatemeh Rezaeipour, Yazd Cardiovascular Research Center, Shahid Sadoughi University of Medical Sciences, Yazd, IR Iran. Tel: +98-3535231421, Fax: \\ +98-3535253335. E-mail: f.rno1@yahoo.com
}

Received 2016 February 26; Revised 2016 May 15; Accepted 2016 June 09.

\begin{abstract}
Background: The arterial ductus is a major communicative pathway which is naturally patent in the fetus, connecting the body of the major pulmonary artery to the descending aorta. Although usually closing on its own, the patent ductus arteriosus (PDA) may remain open in the second postnatal week due to a lack of prompt diagnosis in the initial days of life or an absence of prompt treatment.

Objectives: To prevent the untoward sequelae of patency of the ductus arteriosus, and to avoid invasive surgery at higher ages, the researchers in the present study embarked on determining the effects of oral ibuprofen during the second postnatal week on newborns with patent ductus arteriosus.

Patients and Methods: In this study, 70 neonates aged eight to 14 days, presenting at Khatam-al-Anbia clinic and the NICU ward of Shahid Sadoughi hospital in Yazd, Iran, who were diagnosed with PDA through auscultation of heart murmurs and echocardiography, were randomly assigned to two groups. The experimental group received oral ibuprofen of $10 \mathrm{mg} / \mathrm{kg}$ in day $1,5 \mathrm{mg} / \mathrm{kg}$ in day 2 , and $5 \mathrm{mg} / \mathrm{kg}$ in day 3 administered by their parents. The control group did not receive any drug. Parents were informed of the potential drug complications and side effects and asked to report them to the researchers if any occurred.

Results: After intervention, the patent ductus arteriosus was closed in 62.9\% of the neonates in the experimental group (35 newborns) who received oral ibuprofen, while it was closed in $54.3 \%$ of the control neonates (35 newborns) who did not receive any $\operatorname{drug}(\mathrm{P}=0.628)$. No complications were observed in either of the neonatal groups.

Conclusions: Our findings showed that administration of oral ibuprofen had no significant effect on the medicinal closure of PDA in full-term neonates during the second postnatal week.
\end{abstract}

Keywords: Patent Ductus Arteriosus, Full-Term Neonates, Oral Ibuprofen

\section{Background}

The ductus arteriosus is a large communication pathway that is naturally patent in the fetus, connecting the trunk of the major pulmonary artery to the descending aorta. In full-term neonates, the functional closure of the ductus arteriosus occurs during the first few hours after birth. In most full-term newborns, no blood actually passes through the ductus after 96 hours (1). It has been shown that the increased pressure of the arterial blood oxygen, which occurs following postnatal pulmonary ventilation, leads to the closure of the ductus arteriosus (2). Furthermore, other factors contributing to the closure of the ductus include nitric oxide and proteins. The factors contributing to the dilation of the ductus are hypoxemia, endothelin-1, acetylcholine, bradykinin, norepinephrine, and prostaglandin E1 $(3,4)$. In addition, high altitude is another factor that may delay closure of the PDA (5).
During the initial postnatal hours, any factor that induces a reduction in blood oxygen level or an increase in prostaglandins may impair the course of the closure of the ductus (6). On the other hand, the effects of these substances are related to the intrauterine gestational age of the fetus. In preterm neonates, the susceptibility of the ductus to the effects of patency due to prostaglandins is more noticeable and decreases with increasing age. Hence, patency is more prevalent in premature newborns (7). Moreover, the ductus arteriosus constricts to close itself during the initial postnatal days in full-term neonates (GA $\geq 37$ )(3).

Closure of the PDA is important for the treatment and management of neonates' cardiovascular and respiratory problems (8). The prevalence of PDA in full-term neonates varies between $1 / 2000$ to $1 / 2500$ (9). It has been associated with high morbidity and mortality rates (10). Indomethacin was the first drug used for PDA closure. How- 
ever, since it had many complications of a high prevalence, attempts have been made to replace it with a better drug. Finally, the American Food and Drug Administration granted permission for the IV use of ibuprofen lysine (neoprofen) in cases of PDA in neonates (11).

The effects of ibuprofen on the closure of PDA are similar to those of indomethacin (12). However, it is superior to indomethacin in that it decreases the mesenteric and renal blood circulation to a lesser degree compared to indomethacin (11) and it is accompanied by fewer renal complications (13). Ibuprofen is very effective in the closure of PDA in the initial postnatal days (14). What is important, though, is that the PDA may not yet be discovered due to lack of early diagnosis in the initial postnatal days or lack of prompt management in the second postnatal week.

\section{Objectives}

Consequently, to ward off the untoward complications of PDA and to avoid invasive surgery at higher ages due to aggravation of the symptoms, the researchers in the present study embarked on determining the effects of oral ibuprofen during the second postnatal week on newborns with PDA.

\section{Patients and Methods}

This randomized single-blind clinical trial was carried out on 70 neonates in their first eight to 14 days of life at gestational week 37 who had presented to Khatam-al-Anbia clinic and the NICU ward of Shahid Sadoughi hospital in Yazd, Iran, during 2011 to 2015. The PDA in the neonates was diagnosed during clinical consultation via auscultation of cardiac souffle and confirmed with echocardiography. The cases of PDA with a diameter less than $3 \mathrm{~mm}$ (small size) were included in the study. The exclusion criteria were: ductal dependent disease (especially severe juxtaductal coarctation), cyanotic cardiac diseases including Fallot's tetralogy, severe pulmonary stenosis, and transposition of the great arteries (TGA). Echocardiography was performed on all neonates under study for confirmation of diagnosis before intervention, and for comparison of the case and control groups before and after intervention.

Using the of of Random Numbers, the newborns were randomly assigned into two equal groups of 35: the experimental group, i.e., Group A, who received treatment, and the control group, i.e., Group B, who received nothing. Information pertaining to the neonate's disorder and the therapeutic method used, as well as the untoward complications of ibuprofen, was given to the parents before intervention. Informed written consent was obtained from all parents. The neonates in group A (19 girls and 16 boys) received oral ibuprofen for three consecutive days for the closure of the PDA. The dosage regimen included $10 \mathrm{mg} / \mathrm{kg}$ in day $1,5 \mathrm{mg} / \mathrm{kg}$ in day 2 , and $5 \mathrm{mg} / \mathrm{kg}$ in day 3, which were consecutively administered after 24-hour intervals. The oral ibuprofen syrup consisted of $100 \mathrm{mg} / \mathrm{mL}$ (Ibukim) manufactured by Hakim Pharmaceutics Company, Tehran, and was given to the neonates by their mothers using similar syringes. The neonates in group $B$, or the control group (25 girls and 10 boys), received no drugs in the course of the intervention. The final echocardiography was performed on all neonates in group A and group B at the end of the third week (postnatal day 21). None of the neonates showed any probable complications due to ibuprofen, and the parents expressed no complaints in this regard. Therefore, no neonate suffered from any of the possible complications during the intervention. As a result, there was no patient attrition, and all 70 (100\%) neonates were present in the study population until the end of the study. The gleaned data were analyzed using SPSS software with a chi-squared test $\left(\chi^{2}\right)$, a Fisher's exact test, and a CochranMantel-Haenszel test. The significant $\mathrm{P}$ value was set at 0.05 .

\section{Results}

Our population study consisted of 70 neonates including 44 (62.9\%) girls and 26 (37.1\%) boys with PDA of a diameter less than $3 \mathrm{~mm}$ (small size) who were in their second postnatal week, i.e., within eight to 14 days of life. The experimental group consisted of 35 neonates, including 16 boys and 19 girls, who were treated with oral ibuprofen. The control group consisted of 35 neonates, including 10 boys and 25 girls, who did not receive ibuprofen. The mean weight at the time of admission was $3.68 \mathrm{~kg}$ for the experimental group and $3.32 \mathrm{~kg}$ for the control group. The mean duct size was $2 \mathrm{~mm}$ and $2.12 \mathrm{~mm}$ for the experimental group and the control group, respectively. The mean age for the intervention group was 11.25 days, and the mean age for the control group was 10.77 days.

The neonates' ages ranged from eight to 14 days. Of the 70 neonates with PDA, the ductus was closed in 41 infants of whom 22 were in the experimental group who received oral ibuprofen, and 19 were in the control group who did not receive this drug (Table 1$)$. None of the neonates $(0 \%)$ in either group showed any complications due to ibuprofen, and all of the neonates (100\%) participated in the study until the end. There was no statistically significant difference between the two groups regarding their responses to treatment. The PDA was closed in $62.9 \%$ of the neonates in the experimental (case) group. Also, the PDA was closed in $54.3 \%$ of the neonates in the control group who received 
no oral ibuprofen. Fisher's exact (2-sided) test with a Pvalue $=0.628$ was used to analyze the data.

Table 1. Description of the Effects of Oral Ibuprofen on Medical Closure of Patent Ductus Arteriosus Between Groups ${ }^{\mathrm{a}}$

\begin{tabular}{lccc}
\hline Groups PDA Closure & Experimental Group & Control Group & Total \\
\hline Yes & 22 & 19 & 41 \\
No & 13 & 16 & 29 \\
Total & 35 & 35 & 70 \\
\hline
\end{tabular}

${ }^{\mathrm{a}} \mathrm{P}=0.628$.

Another analysis, in which the boys and girls were separated, revealed that $62.5 \%$ of the boys receiving ibuprofen had closed PDA while $50 \%$ of the boys not receiving ibuprofen had closed PDA. Moreover, 63.2\% of the girls receiving ibuprofen had closed PDA while $56 \%$ of the girls not receiving ibuprofen had closed PDA. This indicated that, as observed in the table, there was no significant difference between groups of either boys $(\mathrm{P}=0.689)$ or girls $(\mathrm{P}=0.76)$ with or without ibuprofen.

To remove the effects of sex, a Cochran-MantelHaenszel test was used with a $\mathrm{P}=0.447$. Having removed the effects of sex, it was revealed that ibuprofen can lead to the closure of PDA by 1.456 fold (3.845 - 0.551). Still, this difference was not significant $(\mathrm{P}=0.448)$. Then, using a logistic model, the variables of age, sex, and ibuprofen consumption were analyzed together. Again, the difference was not found to be significant (Table 2).

Table 2. Logistic Regression Analysis Showing That Ibuprofen Has No Significant Effect on

\begin{tabular}{lccc}
\hline Determining Factor & OR & CI 95\% & PValue \\
\hline Ibuprofen & 0.662 & $1.845-0.237$ & 0.430 \\
Weight & 1.096 & $0.486-2.470$ & 0.825 \\
Sex (female) & 1.156 & $3.196-0.418$ & 0.780 \\
\hline
\end{tabular}

Accordingly, the use of oral ibuprofen was shown to have no significant effects on the closure of PDA in neonates in the experimental group, or group A (62.9\%), as compared to the control group, or group B (54.3\%), who were in their second postnatal week. This lack of efficacy was not due to small sample volume, as it was determined that increasing sample volume would have no effect on the results.

\section{Discussion}

The aim of this study was to determine whether ibuprofen is effective for PDA closure in full-term neonates in the second week after birth. Our study showed that PDA was closed in $62.9 \%$ of the neonates in the experimental group (35 newborns) who received oral ibuprofen, while it was closed in $54.3 \%$ of the control neonates (35 newborns) who did not receive any drug $(\mathrm{P}=0.628)$. This effect was independent of sex and weight. No complications were observed in the neonates.

A study by John McIntyre et al. has suggested that indomethacin was effective in the closure of PDA in premature newborns (8). Moreover, studies by Thomas and van Overmeire et al. (12-14) have concluded that ibuprofen was as effective as indomethacin in the closure of PDA in the third postnatal day in pre-term neonates with respiratory distress syndrome (RDS) with less renal complications Jameii et al. showed that the use of ibuprofen could decrease the serum levels of brain natriuretic peptide type-B (BNP-B) in pre-term neonates with significant PDA (GA $\leq$ $34 \mathrm{w})$, since this peptide increases with cardiac pressure or volume overload; thus, the dropping of the serum level meant a decreasing size of the ventricle due to the decreasing size of the PDA $(15,16)$.

In a study on 68 pre-term neonates with significant PDA, Pistulli et al. (17) compared the effects of oral ibuprofen $(n=36)$ with intravenous ibuprofen $(n=32)$, and found a closure rate of $71.8 \%$ versus $83.3 \%$, respectively; this difference was comparable $(\mathrm{P}=0.35)$. All of the 15 neonates that needed a second course of ibuprofen had clinical signs of infection and positive blood cultures; after a second course of ibuprofen, PDA occluded in $100 \%$ of the neonates. An explanation for this result is that in neonates with infection, the levels of prostaglandins and the tumor necrotizing factor are higher. In Pistulli et al.'s study, ibuprofen was administered in the first week (48 - 96 hours) after birth, and the patients were pre-term. In contrast, the patients in this study were full-term, and we did not use a second course of ibuprofen.

Very few studies have been conducted so far on the efficacy of cyclo-oxygenase inhibitors in the closure of PDA in full-term neonates. In a study by Amoozgar et al. (10) on the effectiveness of oral ibuprofen in the closure of PDA in full-term neonates, the researchers assigned 51 full-term neonates aged three to 30 days (the first month of life) into two groups. The case group including 30 patients who received oral ibuprofen, and the control group including 21 patients who received a placebo for three days. Their findings showed that the PDA was closed in $73.3 \%$ of the neonates in the case group, while it was closed in only $42.9 \%$ of the neonates in the control group; however, they recommended that because of the conditions of their study, more randomized clinical trials were needed to confirm their assertions (10). Regarding their smaller sample size (51 neonates) with a wider age range (the first 
month of life), which can change the results given the influence of varying age factors, our study selected a different course with a more precise statistical procedure. We had 70 neonates as the population to be investigated. Since PDA is not diagnosed in a number of neonates in the first postnatal week, this study was devoted to the full-term neonates in whom PDA was diagnosed in the second postnatal week of life (eight to 14 days). In this way, the time interval was limited, and more accurate results were obtained specifically for those diagnosed in the second week of life.

Again, this study demonstrated that the PDA was ultimately closed in $62.9 \%$ of the full-term newborns who received oral ibuprofen in the second postnatal week, while the ductus was closed in only $54.3 \%$ of the neonates in the control group who received no drugs $(\mathrm{P}=0.628)$. Pourarian et al. (18) used ibuprofen of twofold the present dose (i.e., $20 \mathrm{mg} / \mathrm{kg}$ in day $1,10 \mathrm{mg} / \mathrm{kg}$ in day 2 , and $10 \mathrm{mg} / \mathrm{kg}$ in day 3) for closure of PDA in full-term neonates aged three to 30 days Their study showed that for 18 of the 29 full-term neonates who received this protocol, PDA occluded after four days in $62.1 \%$ versus $43.3 \%$, the latter of which were given the standard dose reported in the study by Amoozgar et al. (10). This means that high doses of ibuprofen are more effective than standard doses of the drug. Also, in the fourth day of treatment, the size of the pulmonic end of the ductus arteriosus decreased from $2.09 \mathrm{~mm}$ to $0.77 \mathrm{~mm}$, compared to $1.68 \mathrm{~mm}$ to $0.81 \mathrm{~mm}$ with the standard dose of oral ibuprofen. Their study evaluated the efficacy of oral ibuprofen for nearly the entire duration of the neonatal period (three to 30 days), while our study evaluated its effects in only the second week (eight to 14 days) of the neonatal period. We limited it in this way because since the serum level of prostaglandin gradually decreases after birth, the patency of the ductus arteriosus is not likely related to the serum level of prostaglandin, and thus ibuprofen (a prostaglandin inhibitor drug) will not be effective. It is, however, important to emphasize that the dose of ibuprofen in their study was twofold that of our study. Perhaps using ibuprofen with higher doses may be more effective in PDA closure, but this must be validated with future studies (18).

The limitations of our study were as follows: first, no measurements of the PDA were taken during the second visits of the neonates, as sedation was needed for careful measurement through echocardiography. Second, a second dose of oral ibuprofen was not administered because the neonates were outpatients, and thus it was not easy accessible to them. Third, it would have been better if there was a third group of neonates who had received a placebo.

\subsection{Conclusion}

Our findings demonstrated that administration of oral ibuprofen in full-term neonates had no significant effect in the closure of PDA in the second postnatal week.

\section{Footnote}

Authors' Contribution: Study concept and design: Mohammad Reza Alipour, Mansooreh Mozaffari Shamsi, and Fatemeh Rezaeipour; analysis and interpretation of data: Mohammad Reza Alipour and Seyedeh Mahdieh Namayandeh; drafting of the manuscript: Mohammad Reza Alipour, Mansooreh Mozaffari Shamsi, Mohammadtaghi Sarebanhassanabadi, and Fatemeh Rezaeipour; critical revision of the manuscript for important intellectual content: Mohammad Reza Alipour, Mohammadtaghi Sarebanhassanabadi, and Zohreh Pezeshkpour; statistical analysis: Mohammad Reza Alipour and Seyedeh Mahdieh Namayandeh.

\section{References}

1. Swartz EN. Is indomethacin or ibuprofen better for medical closure of the patent ductus arteriosus?. Arch Dis Child. 2003;88(12):1134-5. [PubMed: 14670792].

2. Lago P, Bettiol T, Salvadori S, Pitassi I, Vianello A, Chiandetti L, et al. Safety and efficacy of ibuprofen versus indomethacin in preterm infants treated for patent ductus arteriosus: a randomised controlled trial. Eur J Pediatr. 2002;161(4):202-7. [PubMed: 12014386].

3. Allen HD, Driscoll DJ, Shaddy RE, Feltes TF. Moss and Adams' Heart Disease in Infants, Children, and Adolescents: Including the Fetus and Young Adult. Lippincott Williams \& Wilkins; 2013.

4. AM R. The ductus arteriosus and persistent patency of the ductus arteriosus. 3 ed. UK: Futura Publishing House; 2001.

5. Alzamora-Castro V, Battilana G, Abugattas R, Sialer S. Patent ductus arteriosus and high altitude. Am J Cardiol. 1960;5:761-3. [PubMed: 13793062].

6. Heyman E, Morag I, Batash D, Keidar R, Baram S, Berkovitch M. Closure of patent ductus arteriosus with oral ibuprofen suspension in premature newborns: a pilot study. Pediatrics. 2003;112(5):e354. [PubMed: 14595076].

7. Supapannachart S, Limrungsikul A, Khowsathit P. Oral ibuprofen and indomethacin for treatment of patent ductus arteriosus in premature infants: a randomized trial at Ramathibodi Hospital. J Med Assoc Thailand. 2002;85(4):S1252-8.

8. McIntyre JOB, van den Anker JN. Indomethacin Treatment of Patent Ductus Arteriosus in Premature Infants. Paediatr Perinat Drug Ther. 2001;4(3):85-91.

9. Mitchell SC, Korones SB, Berendes HW. Congenital heart disease in 56,109 births. Incidence and natural history. Circulation. 1971;43(3):323-32. [PubMed: 5102136].

10. Amoozgar H, Ghodstehrani M, Pishva N. Oral ibuprofen and ductus arteriosus closure in full-term neonates: a prospective case-control study. Pediatr Cardiol. 2010;31(1):40-3. doi: 10.1007/s00246-009-9542y. [PubMed: 19841966].

11. Jones RW, Pickering D. Persistent ductus arteriosus complicating the respiratory distress syndrome. Arch Dis Child. 1977;52(4):274-81. [PubMed: 324406]. 
12. Grosfeld JL, Chaet M, Molinari F, Engle W, Engum SA, West KW, et al. Increased risk of necrotizing enterocolitis in premature infants with patent ductus arteriosus treated with indomethacin. Ann Surg. 1996;224(3):350-5. [PubMed: 8813263] discussion 355-7.

13. Thomas RL, Parker GC, Van Overmeire B, Aranda JV. A meta-analysis of ibuprofen versus indomethacin for closure of patent ductus arteriosus. Eur J Pediatr. 2005;164(3):135-40. doi: 10.1007/s00431-004-1596-5. [PubMed: 15717178].

14. Van Overmeire B, Follens I, Hartmann S, Creten WL, Van Acker KJ. Treatment of patent ductus arteriosus with ibuprofen. Arch Dis Child Fetal Neonatal Ed. 1997;76(3):F179-84. [PubMed: 9175948].

15. Jameii Khosroshahi A, Kianfar A, Mohammadpour Aharanjani B, Sayadpour Zanjani K. Usefulness of serum brain natriuretic Peptide level for screening hemodynamically significant patent ductus arte- riosus in preterm neonates. Iran J Pediatr. 2014;24(6):766-9. [PubMed: 26019784].

16. Maeda K, Tsutamoto T, Wada A, Hisanaga T, Kinoshita M. Plasma brain natriuretic peptide as a biochemical marker of high left ventricular end-diastolic pressure in patients with symptomatic left ventricular dysfunction. Am Heart J. 1998;135(5 Pt 1):825-32. [PubMed: 9588412].

17. Pistulli E, Hamiti A, Buba S, Hoxha A, Kelmendi N, Vyshka G. The Association between Patent Ductus Arteriosus and Perinatal Infection in A Group of Low Birth Weight Preterm Infants. Iran J Pediatr. 2014;24(1):42-8. [PubMed: 25793044].

18. Pourarian S, Rezaie M, Amoozgar H, Shakiba AM, Edraki MR, Mehdizadegan N. High-Dose Oral Ibuprofen in Treatment of Patent Ductus Arteriosus in Full-Term Neonates. Iran J Pediatr. 2015;25(4):e2005. doi: 10.5812/ijp.2005. [PubMed: 26396694]. 